\title{
Reducing the energy cost of human walking using an unpowered exoskeleton
}

\author{
Steven H. Collins ${ }^{*}, 1$, M. Bruce Wiggin ${ }^{*}, 2$, and Gregory S. Sawicki ${ }^{*}, 2$ \\ ${ }^{1}$ Department of Mechanical Engineering, Carnegie Mellon University, 5000 Forbes Avenue, \\ Pittsburgh, PA, 15213, USA. \\ 2Joint Department of Biomedical Engineering, North Carolina State University and the University \\ of North Carolina at Chapel Hill, 911 Oval Drive, Raleigh, NC 27695, USA.
}

\begin{abstract}
With efficiencies derived from evolution, growth and learning, humans are very well-tuned for locomotion ${ }^{1}$. Metabolic energy used during walking can be partially replaced by power input from an exoskeleton ${ }^{2}$, but is it possible to reduce metabolic rate without providing an additional energy source? This would require an improvement in the efficiency of the human-machine system as a whole, and would be remarkable given the apparent optimality of human gait. Here we show that the metabolic rate of human walking can be reduced by an unpowered ankle exoskeleton. We built a lightweight elastic device that acts in parallel with the user's calf muscles, off-loading muscle force and thereby reducing the metabolic energy consumed in contractions. The device uses a mechanical clutch to hold a spring as it is stretched and relaxed by ankle movements when the foot is on the ground, helping to fulfill one function of the calf muscles and Achilles tendon. Unlike muscles, however, the clutch sustains force passively. The exoskeleton consumes no chemical or electrical energy and delivers no net positive mechanical work, yet reduces the metabolic cost of walking by $7.2 \pm$ $2.6 \%$ for healthy human users under natural conditions, comparable to savings with powered devices. Improving upon walking economy in this way is analogous to altering the structure of the body such that it is more energy-effective at walking. While strong natural pressures have already shaped human locomotion, improvements in efficiency are still possible. Much remains to be learned about this seemingly simple behavior.
\end{abstract}

Humans are skilled walkers. Over generations, our bodies have evolved muscular ${ }^{1}$, skeletal $^{3}$ and neural ${ }^{4}$ systems well-suited to locomotion. We learn and embed walking coordination strategies over our lifetimes ${ }^{5}$ and adapt to new locomotor environments in minutes or seconds ${ }^{6}$. We take about 10,000 steps per day ${ }^{7}$, or hundreds of millions of steps in a

Reprints and permissions information is available at www.nature.com/reprints.

Correspondence and requests for materials should be addressed to S.H.C. (stevecollins@cmu.edu) or G.S.S.

(greg_sawicki@ncsu.edu).

All authors contributed equally to this work.

Supplementary Information is linked to the online version of the paper at www.nature.com/nature

Author Contributions G.S.S. and S.H.C. contributed equally to study design and direction; M.B.W., S.H.C. and G.S.S. designed the device; M.B.W. fabricated the device; M.B.W. and G.S.S. conducted human locomotion experiments; M.B.W., S.H.C. and G.S.S. analyzed data; S.H.C., M.B.W. and G.S.S. drafted the manuscript, S.H.C. and G.S.S. edited the manuscript. All authors approved of the final manuscript.

Source data available at: https://www.bme.ncsu.edu/labs/hpl/NaturePassiveExoData/. 
lifetime, exceeding the approximately 10,000 hours of practice thought to be needed to attain expertise ${ }^{8}$ by adulthood. We naturally keep energy expenditure low during walking, choosing, for example, step length ${ }^{9}$ and even arm motions ${ }^{10}$ that minimize energy cost. Nearly any change to the human musculoskeletal system or its pattern of coordination increases metabolic rate. Despite this skill and efficiency, getting about is still expensive. People expend more energy during walking than any other activity of daily life ${ }^{11}$, and fatigue can limit mobility. Herein lies the challenge: reducing the effort of normal walking could garner substantial benefits, but humans are already so energy-effective that making improvements is extremely difficult.

Since at least the 1890 's ${ }^{12}$, engineers have designed machines intended to make walking easier ${ }^{13-15}$. A survey of these designs can be found in the Supplementary Discussion. It is only recently that any attempt at reducing the energy cost of walking with an external device has met with success. The first machine to do so used off-board pneumatic pumps and valves to replace human joint work with exoskeleton work ${ }^{2}$, overcoming the surprisingly tricky challenge of coordinating assistance with the human neuromuscular system. More recently still, a powered and untethered device using similar control strategies succeeded in reducing energy $\operatorname{cost}^{16}$, overcoming the additional challenge of autonomous packaging.

Reducing the energy cost of walking with an unpowered device requires a different approach. Instead of adding a robotic energy source to replace metabolic sources, one must, in a sense, change the human body such that it is more efficient at locomotion (Extended Data Fig. 1). For the task of carrying heavy loads while walking, such improvements have been demonstrated using a spring-mounted backpack ${ }^{17}$ and by training people to balance the weight on their head in just the right way ${ }^{18}$. But is there room for a similar improvement in the already expert task of normal walking?

The possibility of unpowered assistance is made more likely by the fact that level walking at steady speed requires no power input in theory, and therefore all energy used in this activity is, in a sense, wasted. Simulation models with spring-loaded legs illustrate this idea ${ }^{19}$; their springs store and return energy during each step, but no mechanical work is done by actuators, capitalizing on the fact that the kinetic and potential energy of the body remain constant on average. Humans expend metabolic energy during walking in part to restore energy that has been dissipated, in passive motions of soft tissues ${ }^{20}$ for example, but the greatest portion of waste occurs in muscles. Muscles consume metabolic energy to perform positive work, as required by conservation of energy, but they also use metabolic energy to produce force isometrically and to perform negative work ${ }^{21}$. This places a metabolic cost on body weight support ${ }^{22}$ and on holding tendons as they stretch and recoil ${ }^{23}$. By contrast, mechanical clutches require no energy to produce force.

We designed a lightweight exoskeleton that provides some of the functions of the calf muscles and tendons during walking, but uses more efficient structures for those tasks. It has a spring in parallel with the Achilles tendon (Fig. 1a) connected to the leg using a lightweight composite frame with a lever about the ankle joint (Fig. 1b, Extended Data Fig. 2). A mechanical clutch in parallel with the calf muscles engages the spring when the foot is on the ground and disengages it to allow free motion when the foot is in the air (Fig. 1c, 
Supplementary Video 1). This design was inspired by ultrasound imaging studies suggesting clutch-like behavior of muscle fascicles to hold the spring-like Achilles tendon ${ }^{24}$, the recoil of which leads to the largest burst of positive mechanical power at any joint during walking. The exoskeleton clutch, described in detail in the Supplementary Methods and Supplementary Video 2, has no motor, battery or computer control, and weighs $0.057 \mathrm{~kg}$. The entire exoskeleton has a mass of between 0.408 and $0.503 \mathrm{~kg}$ per leg, depending on participant size (Extended Data Tables 1 and 2). Based on simulation studies of walking with elastic ankles ${ }^{19,25}$, we expected an intermediate stiffness to minimize energy cost and performed tests with a range of springs.

We conducted experiments with healthy participants $(\mathrm{N}=9)$ wearing an exoskeleton on each leg while walking at a normal speed $\left(1.25 \mathrm{~m} \cdot \mathrm{s}^{-1}\right)$ on a treadmill. The exoskeleton produced a pattern of torque similar to that produced by the biological ankle, but with lower magnitude (Fig. 2a). This reduced the ankle moment produced by calf muscles (Fig. 2b) and also reduced calf muscle activation, particularly in the soleus (Fig. 2c). Joint angles changed little across conditions (Fig. 2d), confirming that the exoskeleton did not interfere with other normal ankle functions, such as toe clearance during leg swing (60-100\% stride).

The exoskeleton reduced human metabolic energy consumption when using moderatestiffness springs (Fig. 3). Wearing a lightweight exoskeleton on each ankle without springs did not measurably increase energy cost compared to normal walking. With increasing spring stiffness, metabolic rate first decreased then increased, supporting the hypothesis that an intermediate stiffness would be optimal. The $180 \mathrm{~N} \cdot \mathrm{m} \cdot \mathrm{rad}^{-1}$ spring reduced the metabolic cost of walking to $2.67 \pm 0.14 \mathrm{~W} \cdot \mathrm{kg}^{-1}$ (mean \pm standard error), down from $2.88 \pm 0.10$ $\mathrm{W} \cdot \mathrm{kg}^{-1}$ for normal walking, a reduction of $7.2 \pm 2.6 \%$ (paired t-test: $\mathrm{p}=0.023$ ). Metabolic energy used for walking, or net metabolic rate, is calculated as total metabolic rate minus the rate for quiet standing, which was $1.47 \pm 0.1 \mathrm{~W} \cdot \mathrm{kg}^{-1}$ in this study. The observed reduction is similar to improvements with high-powered devices ${ }^{2,16}$ and equivalent to the effect of taking off a $4 \mathrm{~kg}$ backpack for an average person ${ }^{26}$.

It is difficult to attribute changes in whole-body metabolic rate to a particular change in muscle mechanics ${ }^{27}$, but with this device there is an association with reduced muscle forces at the assisted ankle joints. Muscles consume energy whenever active, even when producing force without performing mechanical work. Simply reducing muscle force can therefore save metabolic energy. For all exoskeleton springs, we measured reductions in the biological component of ankle moment and the activity of major plantarflexor muscles, both indicative of reduced force. Reductions occurred primarily during early and mid-stance (0-40\% stride, Fig. 2b,c) when muscle fascicles are nearly isometric and therefore perform little mechanical work $^{24}$. Simulation models estimate that plantarflexor muscle energy use primarily occurs during this period and accounts for about $27 \%$ of the metabolic energy used for walking 27 . With the $180 \mathrm{~N} \cdot \mathrm{m} \cdot \mathrm{rad}^{-1}$ spring, the biological component of average ankle moment was reduced by $14 \%$ and mid-stance soleus electrical activity was reduced by $22 \%$ compared to normal walking. Extrapolating from these values, one might expect about a $4 \%$ to $6 \%$ reduction in overall metabolic rate, comparable to the observed $7 \%$ reduction. 
Biological contributions to ankle joint work were also partly replaced by the exoskeleton, but it is unlikely that these changes were responsible for reductions in metabolic rate. The connections between joint work, musculotendon work, muscle fascicle work, and metabolic rate are complex. Much of the mechanical work at the ankle joint during walking is the result of elastic stretch and recoil of the Achilles tendon ${ }^{24}$, which does not directly consume metabolic energy. Because of tendon compliance, using an exoskeleton to reduce cyclic musculotendon work can actually preserve or increase the mechanical work performed by muscle fascicles ${ }^{28}$ - reducing tendon force reduces its stretch, which can lead to increased excursion of the muscle itself and more muscle work. Even if reduced joint work had been the result of reduced muscle fascicle work, under these circumstances such a change would likely not have reduced metabolic cost. It has recently been shown that for contraction cycles similar to those of the calf muscles during normal walking, where muscle fascicles undergo stretch-shorten cycles with nearly zero net work, making equal and opposite changes to both negative and positive work has no effect on metabolic energy use per unit force ${ }^{29}$. Our understanding of the relationship between muscle activity and metabolic rate remains imperfect, but reduced muscle work does not seem to provide a good explanation for reduced metabolic cost in this study.

Metabolic rate increased back to normal levels when using high-stiffness exoskeleton springs, apparently the result of several factors. Humans tend to select coordination patterns with similar net ankle moments across a range of exoskeleton torques ${ }^{2,30}$, a trend also observed here. With stiff springs, tibialis anterior activity counteracting exoskeleton torque in early and mid-stance appeared to increase, possibly reducing changes in total joint moment. Knee muscle activity to prevent hyperextension during mid- and late stance may also have contributed to increases in metabolic cost. Unexpectedly, some of the increase in metabolic rate appears to be associated with increased plantarflexor activity at the end of stance. Furthermore, despite being more active during this period, plantarflexor muscles produced lower joint moments. These reduced moments likely reflect increased contraction velocity, because muscle force drops rapidly as the rate of shortening increases. These two observations suggest that exoskeleton support during mid-stance led to inefficient, rapid shortening of plantarflexor muscles during the usual burst of positive work at the end of the step. Also unexpectedly, it does not appear that the increase in metabolic rate with highstiffness springs is well explained by simple dynamic models of walking, which predict changes in center-of-mass work that were not observed here ${ }^{19,25}$. These and other interpretations are presented in expanded form in the Supplementary Discussion and can be explored using joint mechanics, muscle activity and center-of-mass mechanics data presented in Extended Data Figs. 3-8.

The complexity of the neuromuscular system can impede useful application of simple ideas from mechanics and robotics to human locomotion. For example, it is tempting to equate joint work or center-of-mass work with metabolic energy use. However, the benefits derived from reduced muscle activity with this unpowered exoskeleton would not have been discovered using joint-level power estimates as a guide, since these draw attention toward terminal stance and away from early and mid-stance when joint power is negative and of low magnitude. The increased metabolic rate at higher exoskeleton spring stiffness found 
here also cannot be explained using mechanical power, because human contributions decreased or remained suppressed with increasing stiffness. The complex neuromuscular factors underlying these changes make effective integration of assistive devices very challenging and may explain why the threshold of reducing the metabolic rate of normal walking, with ${ }^{2,16}$ or without additional power input, has taken more than a century to cross. Much remains to be learned about human coordination, even in this seemingly uncomplicated activity.

We have demonstrated that net energy input is not a fundamental requirement for reducing the metabolic cost of human walking. Reducing calf muscle forces - while also fulfilling normal ankle functions and minimizing penalties associated with added mass or restricted motions - can provide a benefit. Passive clutch-like structures are feasible in nature, making the use of this type of device analogous to a change in anatomy that improves walking economy. Similar morphological changes might augment other lower-limb musculature or locomotion in other animals. While evolution, growth and learning have driven efficiency, improvements are yet possible.

\section{Methods}

\section{Participants}

Nine healthy adults $(\mathrm{N}=9,2$ female, 7 male; age $=23.0 \pm 3.7$ yrs.; mass $=77.4 \pm 9.2 \mathrm{~kg}$; height $=1.84 \pm 0.10 \mathrm{~m}$; mean \pm s.d.) participated in the study. One additional subject dropped out before completing the protocol, in part due to hardware malfunctions during training sessions. Sample size was chosen based on metabolic rate data from previous studies. All subjects provided written informed consent prior to participation. The study protocol was approved and overseen by the Institutional Review Board of the University of North Carolina at Chapel Hill.

\section{Exoskeleton hardware}

Custom frames were fabricated for each participant using modified orthotics methods. A flexible cast was used to create a positive plaster mold of the foot, ankle and shank, upon which a thin, selectively-reinforced carbon fiber frame was formed. Shank and foot segments were removed from the mold and connected using an aluminum hinge joint with a plain bearing (Extended Data Fig. 2). The custom mechanical clutch ${ }^{31,32}$ (Fig. 1b, Supplementary Methods) was then integrated with the frame. Part drawings and CAD files are provided as Supplementary Data 1 and 2, a detailed accounting of component mass and comparisons to other systems are provided in Extended Data Tables 1 and 2, and a demonstration of clutch function can be found in Supplementary Video 2.

We used five sets of steel coil extension springs with stiffness of 5.6, 7.9, 10.5, 13.3 and $17.2 \mathrm{kN} \cdot \mathrm{m}^{-1}$ and mass of $0.059,0.061,0.068,0.092$ and $0.098 \mathrm{~kg}$, respectively. Spring stiffnesses were determined in experiments where springs were stretched to several displacements using a fixture and forces were measured using a load cell. Springs were attached to a lever arm on the foot frame with an average radius of $0.152 \mathrm{~m}$, resulting in average exoskeleton rotational stiffnesses of $130,180,240,310$ and $400 \mathrm{~N} \cdot \mathrm{m} \cdot \mathrm{rad}^{-1}$. This 
spans the range of reported ankle joint quasi-stiffnesses for walking ${ }^{33}$. To measure force, a single-axis load cell (LC8125-312-500, Omega Engineering Inc., Stamford, CT, USA) was placed in series with the spring. Exoskeleton joint torque was calculated as the product of spring force and the lever arm, assuming constant leverage.

The effective stiffness experienced by participants was lower than that indicated by the springs themselves. In a follow-up experiment with a single subject, quasi-static loading of the exoskeleton, and additional markers on the exoskeleton frame, compliance in the frame and rope led to about an $18 \%$ decrease in effective stiffness, while compliance at the humanexoskeleton interface led to an additional decrease of about $15 \%$. The effective mechanical stiffness of the exoskeleton, when clutched, was therefore likely about $33 \%$ lower than indicated by the springs alone. Such effects likely varied across subjects, being dependent on both frame construction and individual human characteristics.

\section{Walking trials}

Subjects walked on a treadmill at $1.25 \mathrm{~m} \cdot \mathrm{s}^{-1}$ under seven conditions: normal walking without the exoskeleton (No Exoskeleton, No Exo. or NE), walking with the complete exoskeleton but no spring connected (No Spring or $\mathrm{k}=0$ ), and walking with each of the springs attached (exoskeleton spring stiffness $\mathrm{k}=130,180,240,310$ and $400 \mathrm{~N} \cdot \mathrm{m} \cdot \mathrm{rad}^{-1}$ ). In previous studies, humans have taken about 20 minutes to fully adapt to tethered pneumatic ankle exoskeletons ${ }^{34}$. To allow sufficient time for learning, subjects completed 21 minutes of training under each condition over three to four walking sessions prior to data collection. During training, subjects walked under each condition for 7 minutes. Mechanical failure of the clutch occurred for some conditions during some training sessions, resulting in more collection sessions for some subjects, but an equal amount of training (21 minutes) with a functioning exoskeleton for all subjects and conditions. Data were collected during minutes 5-7 of a final 7 minute session, or minutes 26-28 of the multi-day experiment. The order of presentation of conditions was randomized for each subject on the first collection day and then held constant for that subject over the remainder of the experiment. This ensured that each subject's training progress was not confounded by ordering effects. Blinding was not practical in this protocol.

\section{Biomechanics and energetics measurements}

Body segment motions were measured using a reflective marker motion capture system ( 8 T-Series cameras, Vicon, Oxford, UK). Ground reaction forces were measured using a treadmill instrumented with load cells (Bertec, Columbus, OH, USA). Ankle muscle activity (soleus, medial and lateral gastrocnemius, tibialis anterior) was measured using a wired electromyography system (SX230, Biometrics Ltd., Newport, UK). Whole-body oxygen consumption and carbon dioxide production were measured using an indirect calorimetry system (Oxycon Mobile, CareFusion Co., San Diego, CA, USA).

\section{Data analysis}

Joint angles, moments and powers were calculated from body motions and ground reaction forces using inverse kinematics and inverse dynamics analyses ${ }^{35}$ (Visual 3D, C-Motion Inc., Germantown, MD, USA). Components of joint moment and power attributed to the human 
(biological component) were calculated ${ }^{36,37}$ by subtracting the exoskeleton torque or power, measured using onboard sensors, from the total ankle joint moment or power, estimated using inverse dynamics. Center-of-mass power was calculated from ground reaction forces using the individual limbs method ${ }^{38}$. Muscle activity was band-pass filtered $(20-460 \mathrm{~Hz})$ in hardware and then conditioned by rectifying and low-pass filtering with a cutoff frequency of $6 \mathrm{~Hz}$ in software. Medial and lateral gastrocnemius signals were combined to simplify analysis and interpretation. Metabolic rate was estimated from average rates of oxygen consumption $\left(\mathrm{VO}_{2}\right)$ and carbon dioxide production $\left(\mathrm{VCO}_{2}\right)$ during the collection window using a standard formula ${ }^{39}$. The metabolic rate during quiet standing was subtracted from gross metabolic rate to get the net value attributable to the energetic demands of walking $2,10,16,22,26$. Net metabolic rate values were then normalized to subject body mass.

Mechanics data and muscle activity from each condition were broken into strides, determined as the period between subsequent heel strikes of a single leg, and an average stride for each subject and condition was obtained. These average strides were used to calculate values of average moment, mechanical power, and muscle activity for each subject and condition. Average moment and power values were calculated as the time integral of moment and power time series data divided by stride period. Positive and negative average joint moments and powers were separated out using time integrals of periods of positive or negative moment or power, respectively. Average net power was calculated as the time integral of power over the whole stride period. Average moment and power values were normalized to subject body mass. Average muscle activity was calculated as the time integral of muscle activity divided by stride period. Average muscle activity during additional periods of interest was calculated as the time integral of muscle activity during those periods divided by stride period (e.g. early and mid-stance, defined as $0-40 \%$ stride, and late stance, defined as $40-60 \%$ stride). Muscle activity was normalized to the maximum value observed during normal walking for each muscle and for each subject. For each condition, study-wide average trajectories of lower-limb joint angles, moments and powers were calculated by averaging across subjects, used for display purposes in Fig. 2 and Extended Data Figs. 3-8.

\section{Statistics}

For each condition, means and standard errors of net metabolic rate, average moment, average mechanical power and average muscle activity outcomes were calculated across subjects, with standard error indicating inter-subject variability. Based on the expectation that user performance would be a non-linear function of exoskeleton stiffness ${ }^{25}$, we conducted a mixed-model, three-factor ANOVA (random effect: subject; fixed effects: spring stiffness and square of spring stiffness) to test for an effect of spring stiffness across exoskeleton conditions (significance level $a=0.05$; JMP Pro, SAS Inc., Cary, NC, USA). For the primary outcome measure, net metabolic rate, stiffness had a significant effect. We used paired t-tests with a Sidak-Holm correction for multiple comparisons ${ }^{40}$ to compare spring conditions to each other and to the No Exoskeleton condition to identify which exoskeleton springs exacted a significant change in metabolic rate. We used a Jarque-Bera two-sided goodness-of-fit test to confirm applicability of tests that assume a normal distribution. For the primary outcome measure, net metabolic rate, we also used a least- 
squares regression to fit a second order polynomial (quadratic) function relating mean outcome data to exoskeleton spring stiffness. Additional two-factor ANOVA analyses (random effect: subject; fixed effect: spring stiffness) were performed to test for an effect of spring stiffness across exoskeleton conditions for secondary outcomes in joint mechanics, center-of-mass mechanics and muscle activity. These results are compiled in Supplementary Table 1.

\section{Extended Data}

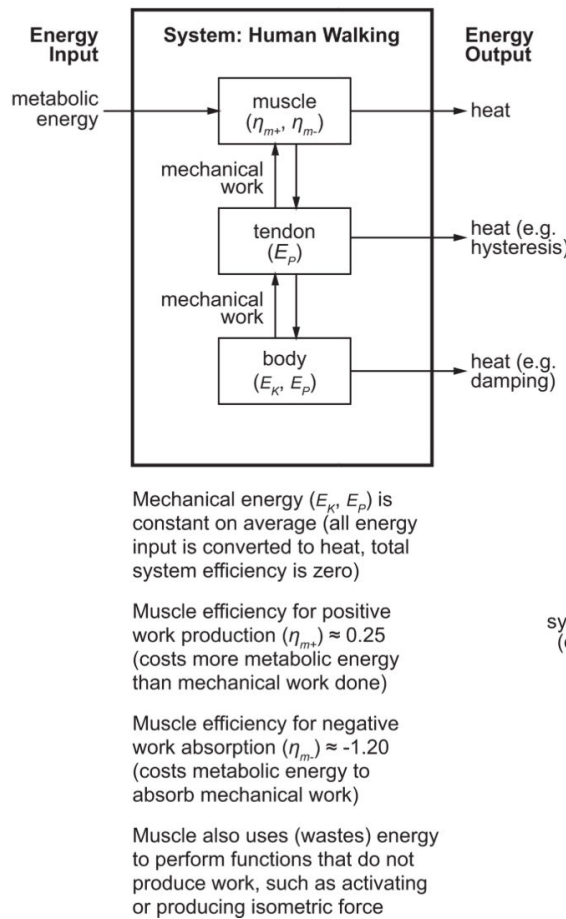

b

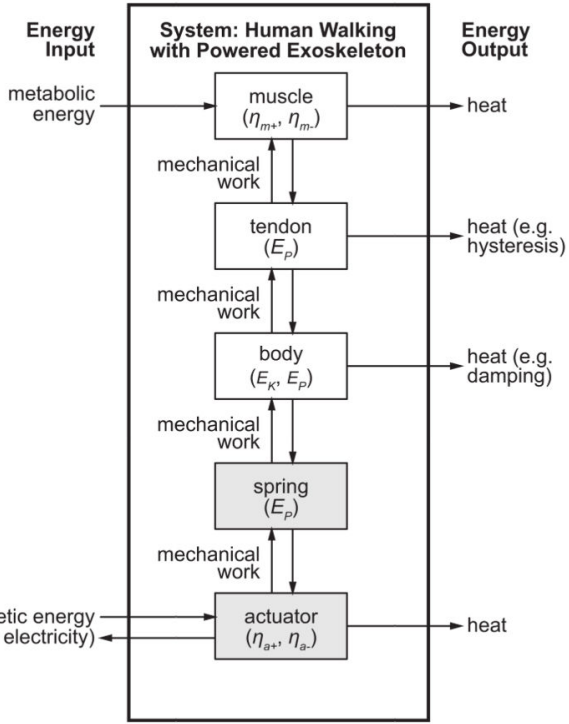

Desired outcome: with electrical energy input, metabolic energy input decreases (total dissipation may increase, system energy is unchanged, overall system energy economy may be worsened)

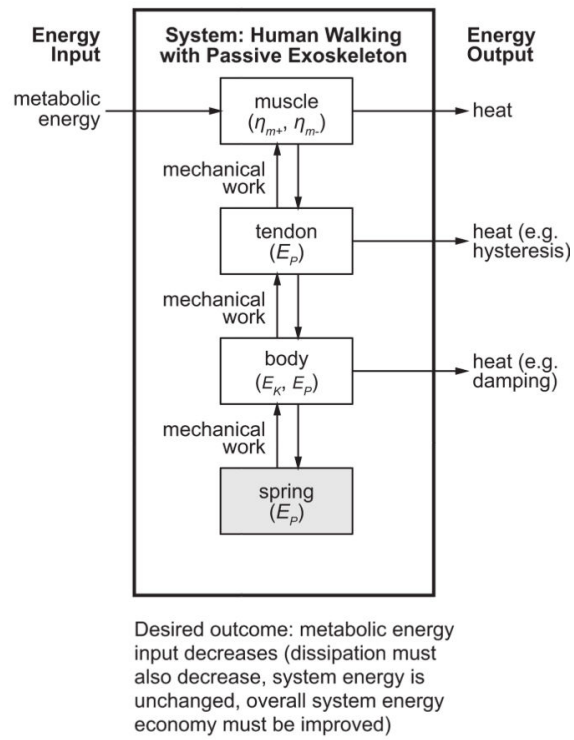

Extended Data Figure 1. Energy diagrams for human-exoskeleton walking Each diagram includes energy inputs, outputs, storage and transfers within the mechanical system, depicted for steady-state walking. In each case, all chemical or electrical energy input is eventually output as heat, since the mechanical energy of the system is constant on average and no useful work is performed on the body or the environment. Energy efficiency, strictly defined, is therefore zero in all cases, and so energy effectiveness or energy economy is instead characterized in terms of 'cost of transport', which is the energy used per unit weight per unit distance traveled ${ }^{41}$. (a) Energy diagram for normal human walking. Muscles consume metabolic energy both to produce mechanical work and to absorb it (and to perform a variety of other functions, such as activating or producing force), and so metabolic energy flows only into the system. Energy loss in muscle manifests as heat. Inside the mechanical system, tendons exchange energy with both the muscle and the body, while kinetic and gravitational potential energy are exchanged within the body segments, all at high mechanical efficiency. Body segment mechanical energy is dissipated only in damping in soft tissues, e.g. during collisions, which is small (about 3\% of the total metabolic energy input $^{20}$ ), and in friction from slipping of the feet against the ground, deformation of the 
ground, or air resistance, all of which are negligible under typical conditions. All of these mechanical losses manifest as heat. (b) Energy diagram for walking with a powered exoskeleton. An additional energy input is provided in the form of, e.g., electricity. The total energy input (and corresponding eventual dissipation) of the system can therefore increase, even if a smaller portion is borne by the human, resulting in poorer overall energy economy. This has been the case with the two powered devices that have reduced the metabolic energy cost of human walking ${ }^{2,16}$. In theory, overall energy economy could still be improved with a powered device in three ways. First, positive mechanical work from muscles could be replaced by work done by a motor with higher efficiency. Second, negative mechanical work could be replaced by generation done by a motor with higher (than -120\%) efficiency, thereby usefully recapturing energy that would otherwise be dissipated as heat. In fact, because muscle expends metabolic energy to absorb mechanical work, it is theoretically possible to simultaneously reduce metabolic rate and capture electrical energy with zero electrical input ${ }^{42}$, although this has yet to be demonstrated in practice. Third, the powered device could approximate an unpowered device, with negligible amounts of electricity used only to control the timing of mechanical elements like clutches ${ }^{43}$. (c) Energy diagram for walking with an unpowered exoskeleton. No additional energy supply is provided and so, unlike the powered case, the only way to decrease metabolic energy use is to reduce total system energy dissipation, or, equivalently, to improve the energy economy of the system as a whole. Note that the only difference from normal human walking, in terms of energy flow, is the addition of elements like springs that store and transfer mechanical energy within the system. In this sense, reducing metabolic rate with a passive exoskeleton is akin to changing the person's morphology such that it is more energy-effective at locomotion. 


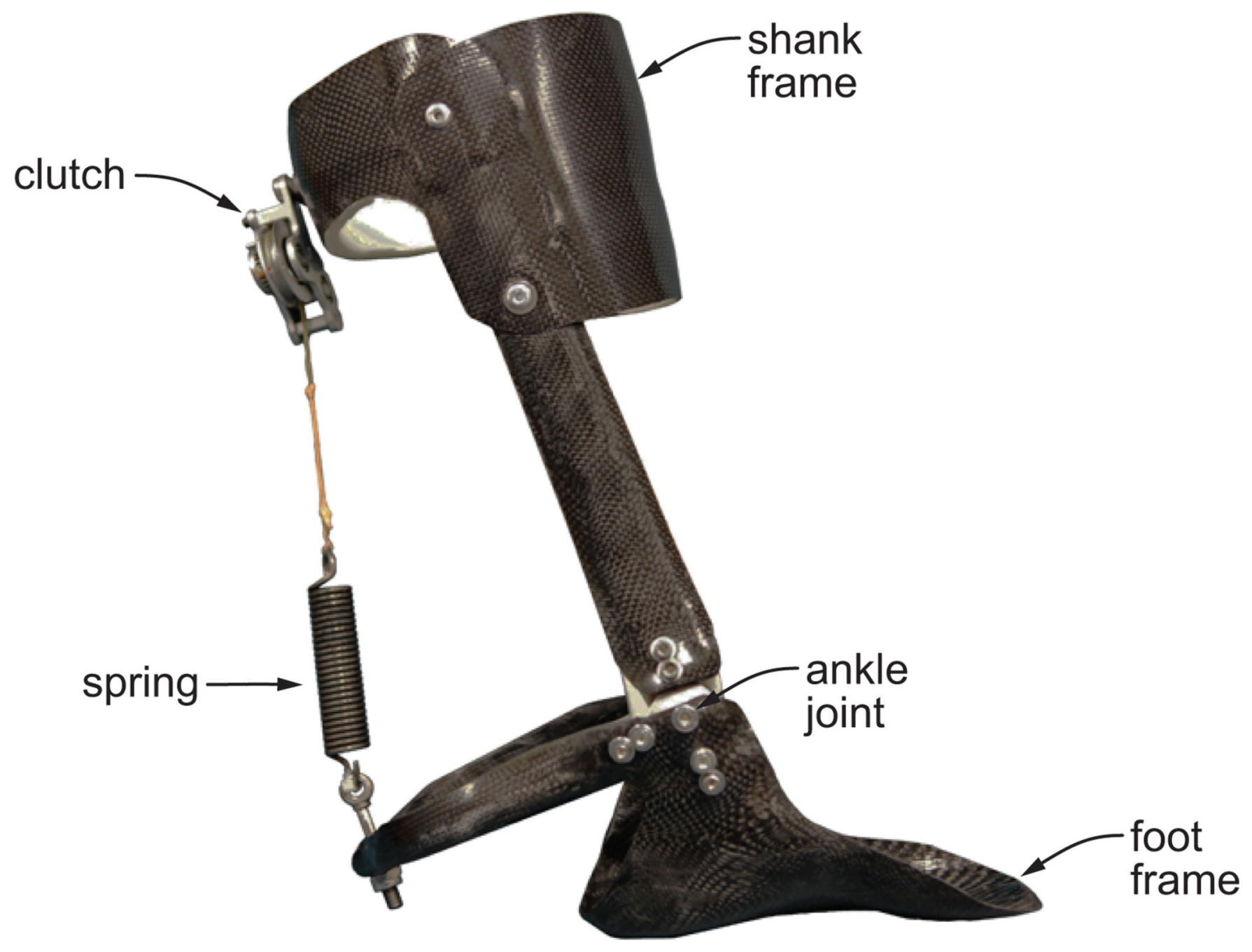

Extended Data Figure 2. Exoskeleton frame design

A rigid carbon fiber shank frame and foot frame were custom made for each participant. The shank section clamps onto the user's lower leg just below the knee and connects to the foot frame through a rotary joint at the ankle. The foot frame includes a lever arm protruding to the rear of the heel, to which the parallel spring is connected. The clutch is mounted to the shank frame posterior to the calf muscles. 

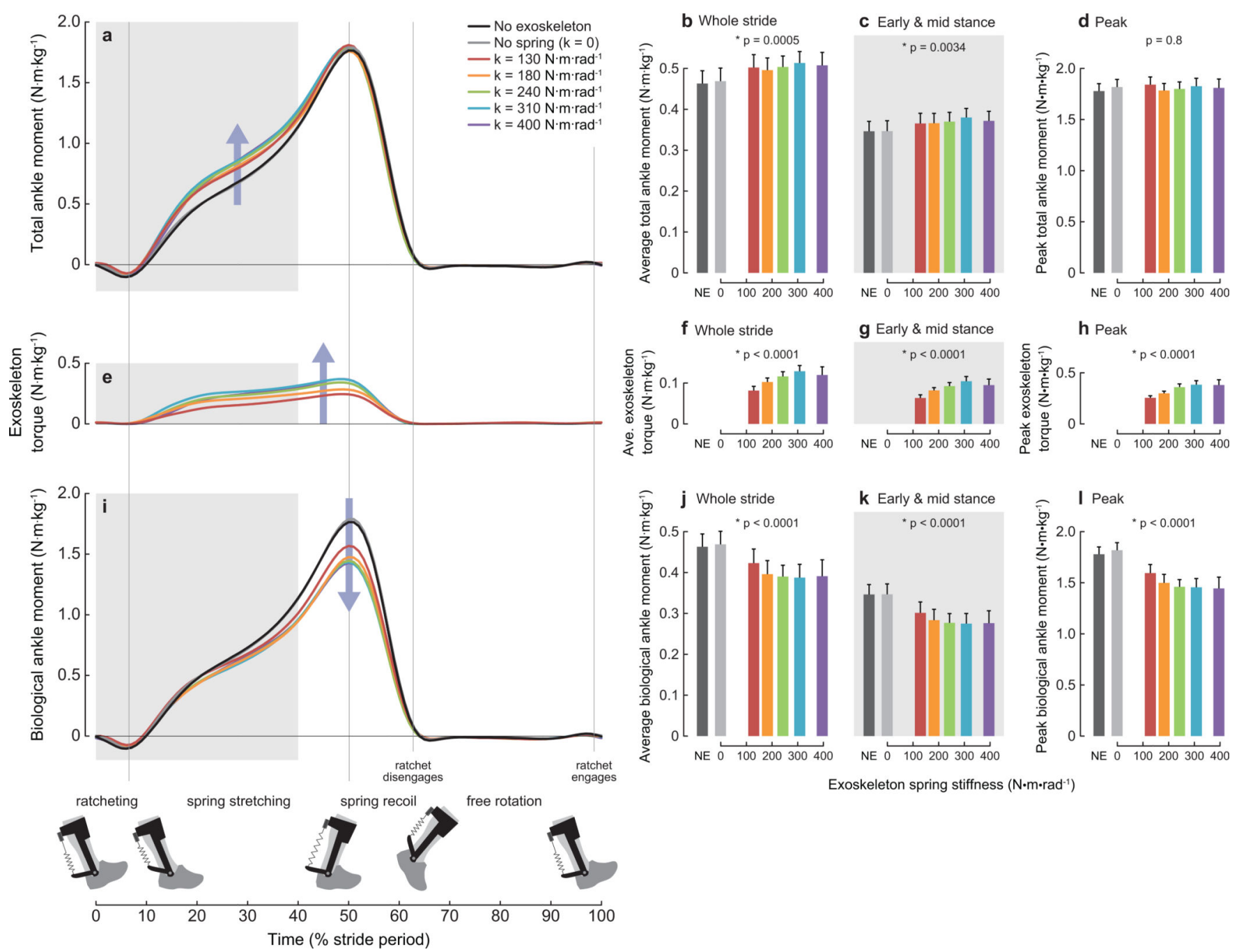

Exoskeleton spring stiffness $\left(\mathrm{N} \cdot \mathrm{m} \cdot \mathrm{rad}^{-1}\right)$

\section{Extended Data Figure 3. Ankle moment contributions}

(a) Total ankle moment, measured using a motion capture system. Average total ankle moment (b) during the entire stride and (c) during early and mid-stance, defined as 0-40\% stride, and (d) peak ankle moment. All spring conditions increased average total joint moment slightly during early stance, but peak total joint moment was maintained across conditions. (e) Exoskeleton torque contribution, as measured using onboard sensors.

Average exoskeleton torque (f) during the entire stride and (g) during early and mid-stance, defined as $0-40 \%$ stride, and (h) peak exoskeleton torque. Average and peak exoskeleton torque increased with increasing exoskeleton spring stiffness, except with the highest stiffness spring. (i) Biological contributions to ankle moment, calculated as the subtraction of the exoskeleton moment from the total moment. Average biological ankle moment (j) during the entire stride and $(\mathrm{k})$ during early and mid-stance, defined as $0-40 \%$ stride, and (l) peak ankle moment. Ankle moments arising from muscle activity decreased with increasing exoskeleton spring stiffness, but with diminishing returns at high spring stiffness. 

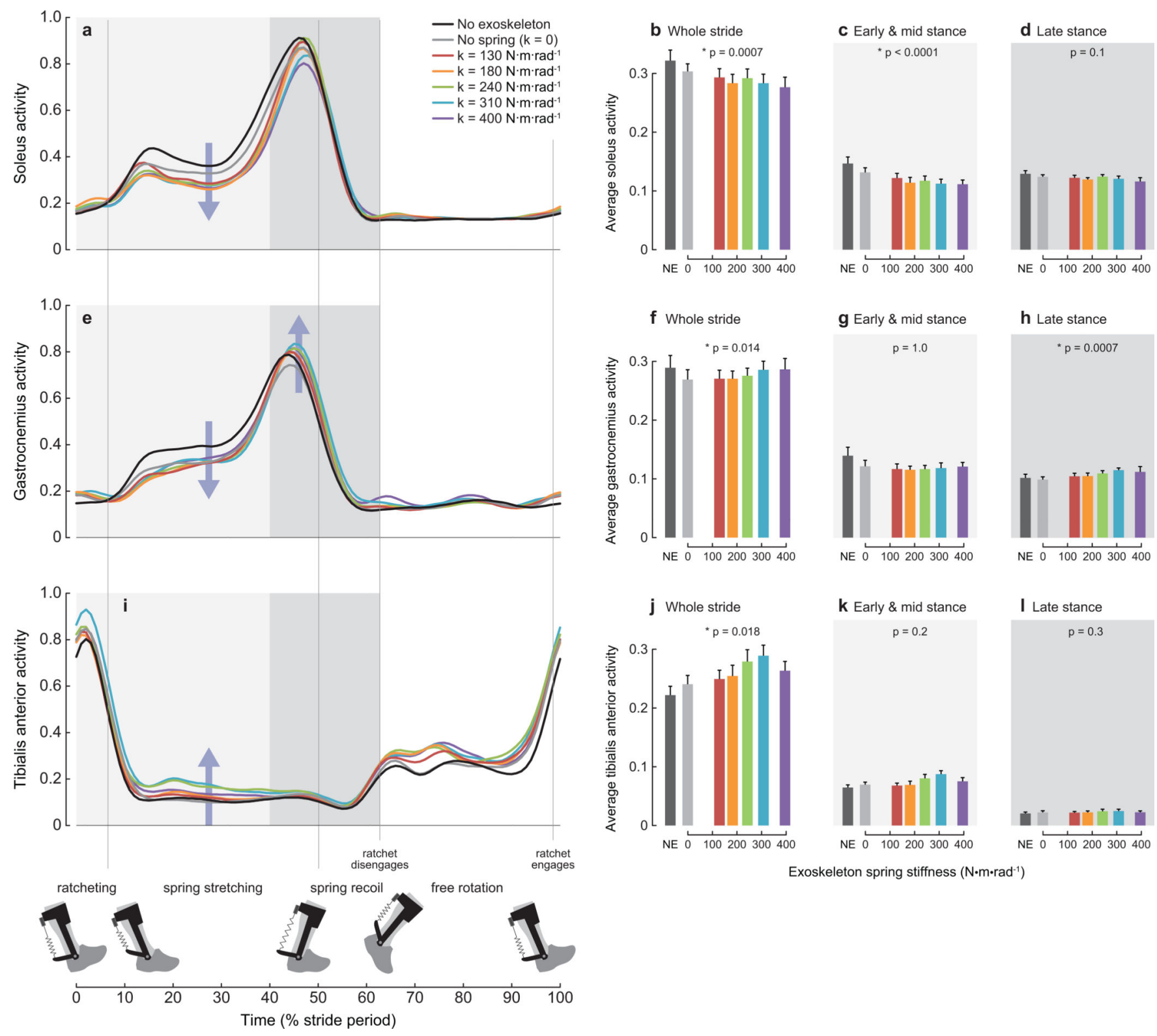

Exoskeleton spring stiffness $\left(\mathrm{N} \cdot \mathrm{m} \cdot \mathrm{rad}^{-1}\right)$

Extended Data Figure 4. Ankle muscle activity

(a) Activity in the soleus, a mono-articular muscle group that acts to plantarflex the ankle. Average soleus activity over (b) the whole stride, (c) early and mid-stance, defined as 0 $40 \%$ stride, and (d) late stance, defined as $40-60 \%$ stride. Soleus activity decreased with increasing spring stiffness. (e) Activity in the gastrocnemius, a biarticular muscle group that acts to plantarflex the ankle and flex the knee. Average gastrocnemius activity over (f) the whole stride, (g) early and mid-stance, defined as $0-40 \%$ stride, and (h) late stance, defined as $40-60 \%$ stride. Gastrocnemius activity was reduced compared to the No Exoskeleton condition during early and mid-stance, but increased with increasing spring stiffness during late stance. (i) Activity in the tibialis anterior, a mono-articular muscle group that acts to dorsiflex the ankle. Average tibialis anterior activity over (j) the whole stride, (k) early and mid-stance, defined as $0-40 \%$ stride, and (1) late stance, defined as $40-60 \%$ stride. Tibialis 
anterior activity seemed to increase during early and mid-stance, and was unchanged during late stance. All values were measured using electromyography and normalized to maximum activity during normal walking.

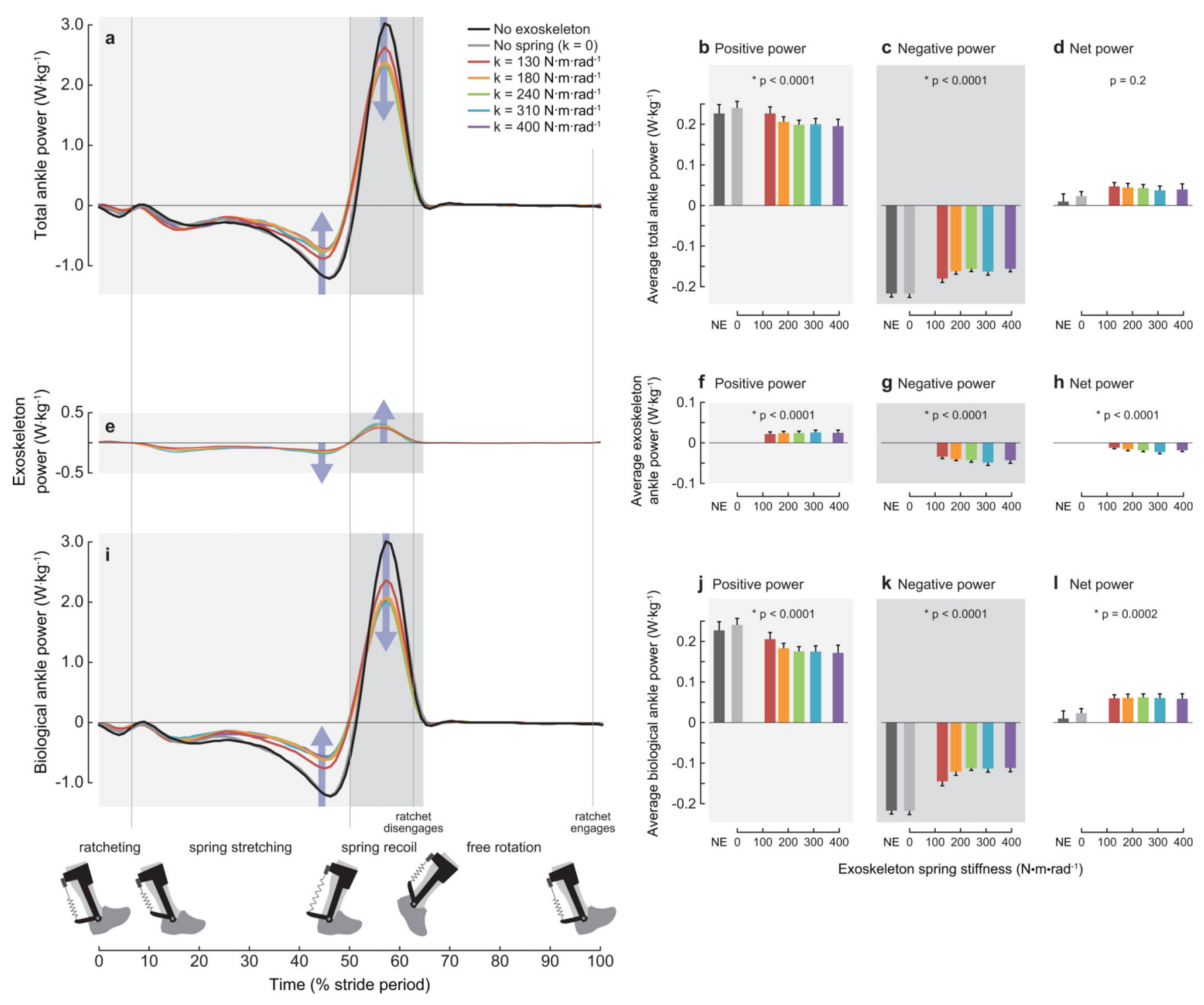

Extended Data Figure 5. Ankle power contributions

(a) Mechanical power of the combined human-exoskeleton system, measured using a motion capture system, (b) average positive power, defined as positive work divided by stride time, (c) average negative power, defined as negative work divided by stride time, and (d) average net power, equivalent to average power, defined as the sum of positive and negative work divided by stride time. Total positive ankle joint power decreased with increasing stiffness, while net joint power increased. (e) Exoskeleton power, measured using onboard sensors for torque and motion capture for joint velocity, (f) average positive exoskeleton power, (g) average negative exoskeleton power, and (h) average net exoskeleton power. Net exoskeleton power was always negative. (i) Biological ankle power, defined as the subtraction of exoskeleton power from total ankle power, ( $\mathrm{j}$ ) average positive biological 
power, (k) average negative biological power, and (l) average net biological power. Net biological power increased with the exoskeleton compared to normal walking.
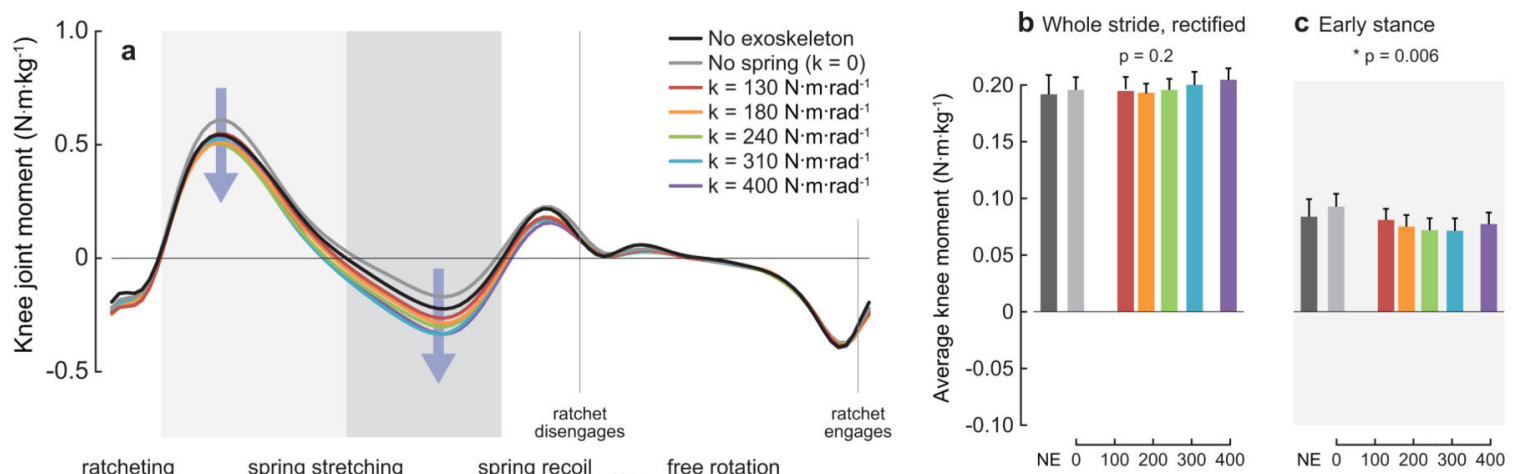

d Late stance ${ }^{*} p<0.0001$
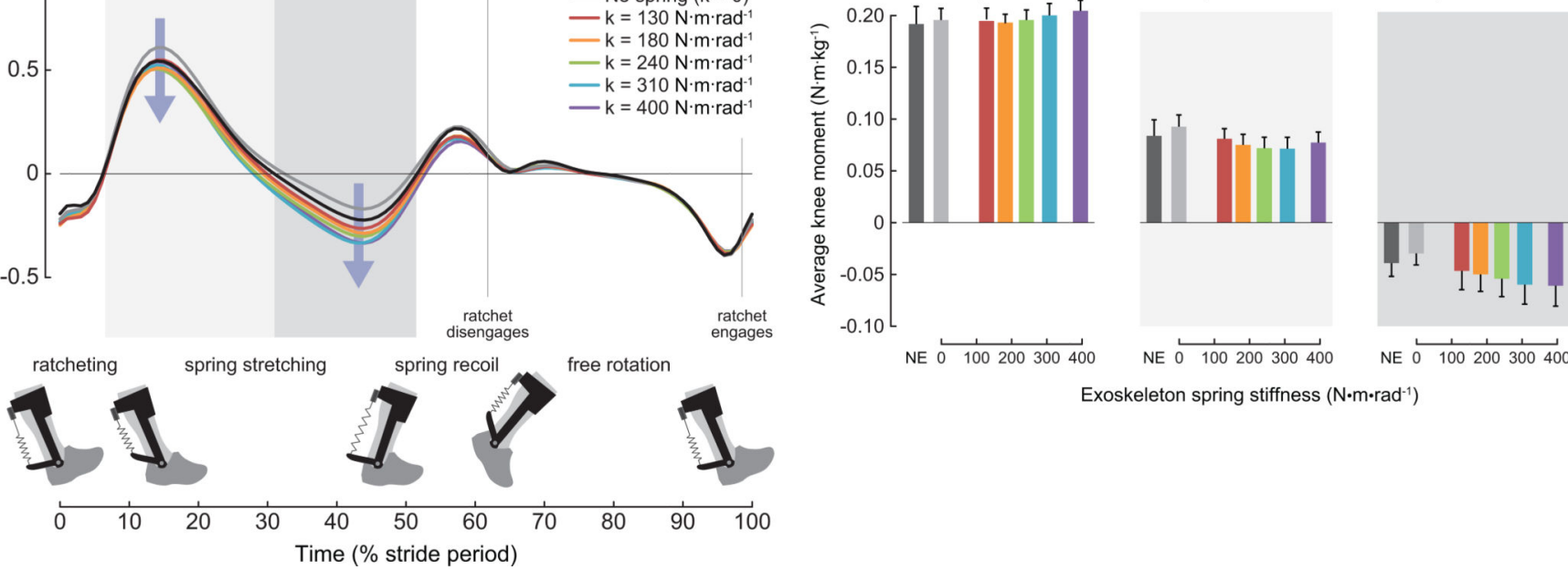

Exoskeleton spring stiffness $\left(\mathrm{N} \cdot \mathrm{m} \cdot \mathrm{rad}^{-1}\right)$

Extended Data Figure 6. Knee moment

(a) Knee moment in time as measured by motion capture, (b) average absolute knee moment over the entire stride, (c) average knee moment during early stance, defined as the positive impulse within approximately 10-30\% stride divided by stride period, (d) average knee moment during late stance, defined as the negative impulse within approximately 30-50\% stride divided by stride period. Average knee moment during late stance increased in magnitude with the highest stiffness springs. Positive values denote knee extension. 

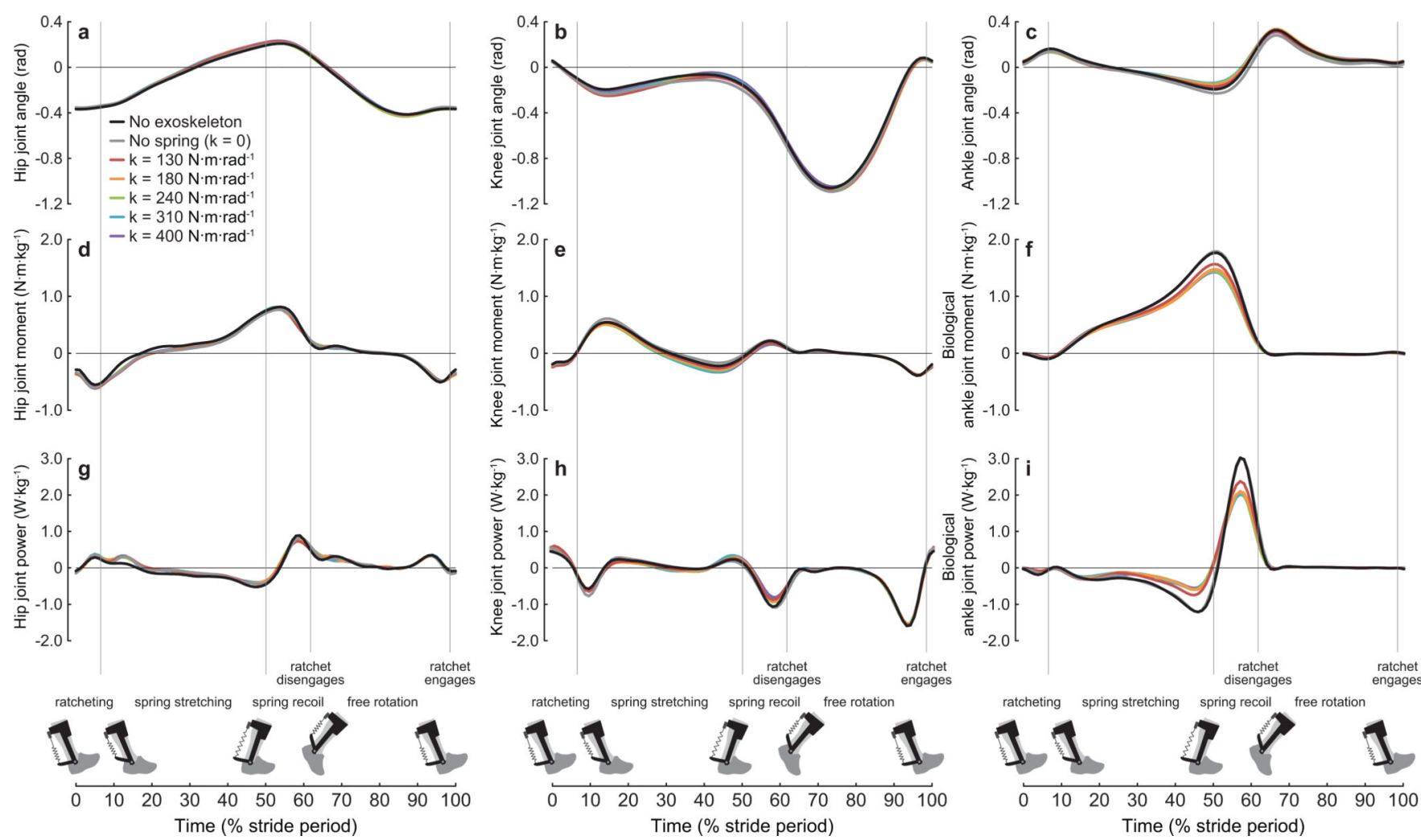

Extended Data Figure 7. Hip, knee and ankle joint mechanics

Joint angles, moments and powers are presented at the same scale to facilitate comparisons across joints. (a) Hip joint angle, (b) knee joint angle, and (c) ankle joint angle. Joint angle trajectories did not appear to change substantially across conditions. (d) Hip moment, (e) knee moment, and (f) biological component of ankle moment. Hip moment did not appear to change substantially across conditions, while knee moment and ankle moment showed trends detailed in Extended Data Figures 6 and 3, respectively. (g) Hip joint power, (h) knee joint power, and (i) the biological component of ankle joint power. Hip and knee power did not appear to change substantially across conditions, while biological ankle power showed trends detailed in Extended Data Figure 5. Positive values denote hip extension, knee extension and ankle plantarflexion with respect to standing posture. 

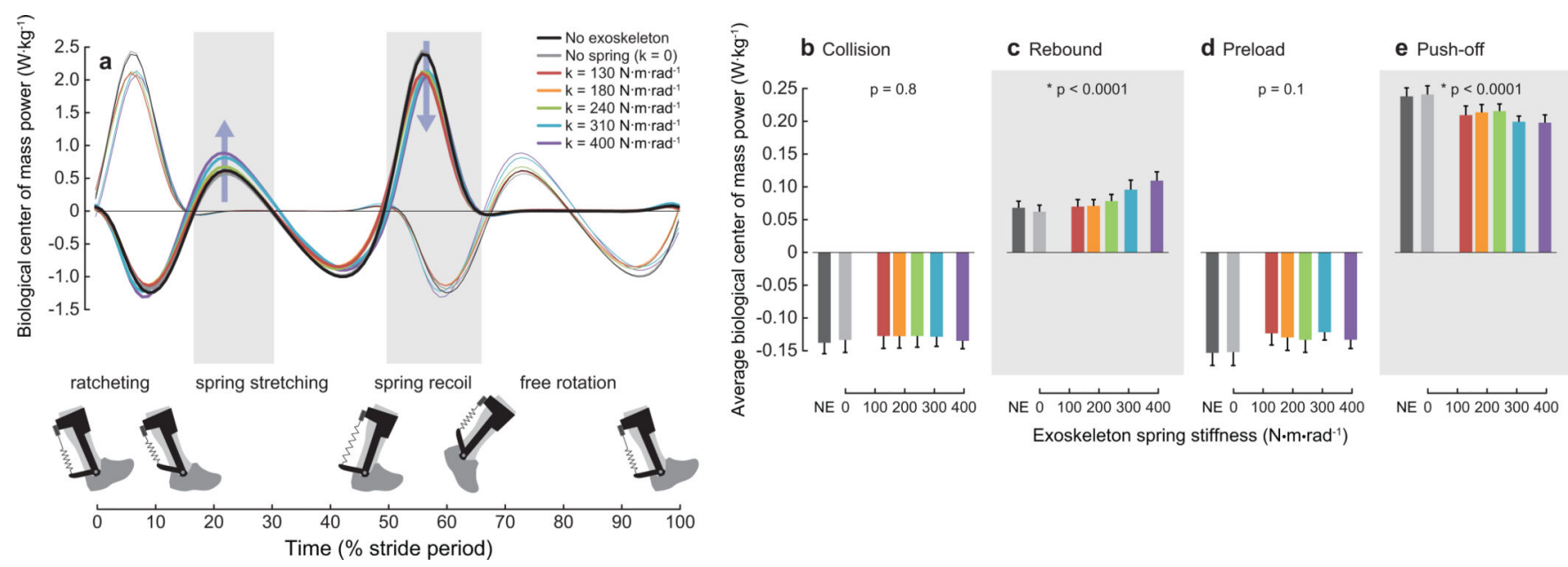

Extended Data Figure 8. Center-of-mass mechanics

(a) The biological contribution to center-of-mass power for each individual limb, defined as the dot product of ground reaction force with center-of-mass velocity, both determined from force plate data, minus the ankle exoskeleton power. (b) Average collision power, defined as the negative work performed during the first half of stance divided by stride time. (c)

Average rebound power, defined as the positive work performed during mid-stance divided by stride time. (d) Average preload power, defined as the negative work performed during mid-stance divided by stride time. (e) Average push-off power, defined as the positive work performed during late stance divided by stride time. With increasing spring stiffness, the human contribution to push-off work decreased, while the human contribution to rebound work increased substantially.

\section{Extended Data Table 1}

Passive ankle exoskeleton mass by component.

\begin{tabular}{ccc}
\hline Segment & US Size & US Size \\
$\mathbf{8}$ & $\mathbf{1 3}$ \\
\hline Carbon Fiber Foot Section & $130 \mathrm{~g}$ & $155 \mathrm{~g}$ \\
Aluminum Ankle Joints (x2) & $40 \mathrm{~g}$ & $40 \mathrm{~g}$ \\
Carbon Fiber Shank Section & $105 \mathrm{~g}$ & $165 \mathrm{~g}$ \\
Frame Mass & $\mathbf{2 7 5 g}$ & $\mathbf{3 6 0 g}$ \\
Average Spring & $60 \mathrm{~g}$ & $60 \mathrm{~g}$ \\
Mechanical Clutch & $57 \mathrm{~g}$ & $57 \mathrm{~g}$ \\
\hline Total Mass & $\mathbf{3 9 2 g}$ & $\mathbf{4 7 7 g}$ \\
\hline
\end{tabular}




\section{Extended Data Table 2}

Comparison of ankle exoskeleton masses.

\begin{tabular}{cc}
\hline Author & $\begin{array}{c}\text { Mass of } \\
\text { Exoskeleton } \\
\text { (grams per leg) }\end{array}$ \\
\hline Mooney et al. ${ }^{10}$ & 2,000 \\
Sawicki et al. ${ }^{9}$ & $1,210^{*}$ \\
Malcolm et al. ${ }^{2}$ & $760^{*}$ \\
Passive Elastic (size 13 US) & 477 \\
Passive Elastic (size 8 US) & 392 \\
\hline * Does not include tethered hardware.
\end{tabular}

\section{Supplementary Material}

Refer to Web version on PubMed Central for supplementary material.

\section{Acknowledgments}

Thanks to A. Ruina, A. Kuo and T. Roberts for detailed editorial suggestions, B. Reich for discussions on statistical analysis, A. Westbrook for data collection, K. Takahashi and R. Nuckols for stiffness characterizations, R. Jackson for data collection and manuscript editing, and J. Caputo, P. Collins, S. Diller, N. Donahue, I. Lau, L. Lau, C. Majidi, J. Malen, A. Robinson, P. Taggart, K. Witte, M. Wu and others for editorial suggestions. Funding for this research provided by grants to G.S.S. from: the NC State Faculty Research and Professional Development Fund; the NC State Chancellors Innovation Fund; Grant \#2011152 from the U.S.-Israel Binational Science Foundation; and Award Number R01NR014756 from the National Institute of Nursing Research of the National Institutes of Health. This material is based upon work supported by the National Science Foundation under Grant No.

IIS-1355716 to S.H.C. The content is solely the responsibility of the authors and does not necessarily represent the official views of the National Institutes of Health or other funding agencies listed.

The authors declare competing financial interests: a non-provisional patent that covers the device has been issued (US2013/0046218 A1, Feb. 2013).

\section{References}

1. Alexander, RM. Principles of Animal Locomotion. Princeton, NJ, USA: Princeton University Press; 2003.

2. Malcolm P, Derave W, Galle S, De Clercq D. A simple exoskeleton that assists plantarflexion can reduce the metabolic cost of human walking. PLoS ONE. 2013; 8:e56137. [PubMed: 23418524]

3. Lovejoy CO. The natural history of human gait and posture: Part 1. Spine and pelvis. Gait Posture. 2005; 21:95-112. [PubMed: 15536039]

4. Dietz V. Spinal cord pattern generators for locomotion. Clin. Neurophys. 2003; 114:1379-1389.

5. Forssberg H. Ontogeny of human locomotor control I. Infant stepping, supported locomotion and transition to independent locomotion. Exp. Brain Res. 1985; 57:480-493. [PubMed: 3979491]

6. Davidson PR, Wolpert DM. Widespread access to predictive models in the motor system: a short review. J. Neur. Eng. 2005; 2:S313-S319.

7. Tudor-Locke C, Johnson WD, Katzmarzyk PT. Accelerometer-determined steps per day in US adults. Med. Sci. Sports Exer. 2009; 41:1384-1391.

8. Ericsson KA, Charness N. Expert performance: Its structure and acquisition. Amer. Psychol. 1994; 49:725.

9. Zarrugh MY, Todd FN, Ralston HJ. Optimization of energy expenditure during level walking. Eur. J. Appl. Physiol. Occu. Physiol. 1974; 33:293-306. 
10. Collins SH, Adamczyk PG, Kuo AD. Dynamic arm swinging in human walking. Proc. Roy. Soc. Lon. B. 2009; 276:3679-3688.

11. Westerterp KR. Physical activity and physical activity induced energy expenditure in humans: measurement, determinants, and effects. Front. Physiol. 2013; 4:90. [PubMed: 23637685]

12. Yagn, N. Apparatus for facilitating walking, running and jumping. U.S. Patent No. 420,179. 1890.

13. Grabowski AM, Herr HM. Leg exoskeleton reduces the metabolic cost of human hopping. J. Appl. Physiol. 2009; 107:670-678. [PubMed: 19423835]

14. van Dijk, W.; van der Kooij, H.; Hekman, E. A passive exoskeleton with artificial tendons: design and experimental evaluation; Proc. IEEE Int. Conf. Rehabil. Rob; 2011. p. 5975470

15. Zoss A, Kazerooni H. Design of an electrically actuated lower extremity exoskeleton. Adv. Robot. 2006; 20:967-988.

16. Mooney LM, Rouse EJ, Herr HM. Autonomous exoskeleton reduces metabolic cost of human walking. J. NeuroEng. Rehabil. 2014; 11:1-6. [PubMed: 24393611]

17. Rome LC, Flynn L, Yoo TD. Biomechanics: Rubber bands reduce the cost of carrying loads. Nature. 2006; 444:1023-1024. [PubMed: 17183310]

18. Heglund NC, Willems PA, Penta M, Cavagna GA. Energy-saving gait mechanics with headsupported loads. Nature. 1995; 375:52-54. [PubMed: 7723841]

19. Zelik KE, Huang TWP, Adamczyk PG, Kuo AD. The role of series ankle elasticity in bipedal walking. J. Theor. Biol. 2014; 346:75-85. [PubMed: 24365635]

20. Zelik KE, Kuo AD. Human walking isn't all hard work: evidence of soft tissue contributions to energy dissipation and return. J. Exp. Biol. 2010; 213:4257-4264. [PubMed: 21113007]

21. Ryschon TW, Fowler MD, Wysong RE, Anthony A, Balaban RS. Efficiency of human skeletal muscle in vivo: comparison of isometric, concentric, and eccentric muscle action. J. Appl. Physiol. 1997; 83:867-874. [PubMed: 9292475]

22. Grabowski A, Farley CT, Kram R. Independent metabolic costs of supporting body weight and accelerating body mass during walking. J. Appl. Physiol. 2005; 98:579-583. [PubMed: 15649878]

23. Roberts TJ. The integrated function of muscles and tendons during locomotion. Compar. Biochem. Physiol. Part A. 2002; 133:1087-1099.

24. Ishikawa M, Komi PV, Grey MJ, Lepola V, Bruggemann GP. Muscle-tendon interaction and elastic energy usage in human walking. J. Appl. Physiol. 2005; 99:603-608. [PubMed: 15845776]

25. Bregman DJ, et al. The effect of ankle foot orthosis stiffness on the energy cost of walking: a simulation study. Clin. Biomech. 2011; 26:955-961.

26. Browning RC, Modica JR, Kram R, Goswami A. The effects of adding mass to the legs on the energetics and biomechanics of walking. Med. Sci. Sports Exer. 2007; 39:515.

27. Umberger BR, Rubenson J. Understanding muscle energetics in locomotion: new modeling and experimental approaches. Exerc. Sport Sci. Rev. 2011; 39:59-67. [PubMed: 21206279]

28. Farris DJ, Robertson BD, Sawicki GS. Elastic ankle exoskeletons reduce soleus muscle force but not work in human hopping. J. Appl. Physiol. 2013; 115:579-585. [PubMed: 23788578]

29. Holt NC, Roberts TJ, Askew GN. The energetic benefits of tendon springs in running: is the reduction of muscle work important? J. Exp. Biol. 2014; 217:4365-4371. [PubMed: 25394624]

30. Kao PC, Lewis CL, Ferris DP. Invariant ankle moment patterns when walking with and without a robotic ankle exoskeleton. J. Biomech. 2010; 43:203-209. [PubMed: 19878952]

\section{Methods References}

31. Wiggin, MB.; Collins, SH.; Sawicki, GS. An exoskeleton using controlled energy storage and release to aid ankle propulsion; Proc. IEEE Int. Conf. Rehabil. Robot; 2011. p. 5975342

32. Wiggin, MB.; Sawicki, GS.; Collins, SH. Apparatus and clutch for using controlled storage and release of mechanical energy to aid locomotion. U.S. Patent No. 20,130.046,218. 2013.

33. Shamaei K, Sawicki GS, Dollar AM. Estimation of quasi-stiffness and propulsive work of the human ankle in the stance phase of walking. PLoS ONE. 2013; 8:e59935. [PubMed: 23555839]

34. Galle S, Malcolm P, Derave W, De Clercq D. Adaptation to walking with an exoskeleton that assists ankle extension. Gait \& Posture. 2013; 38:495-499. [PubMed: 23465319] 
35. Winter, DA. Biomechanics and Motor Control of Human Movement. New York, NY, USA: John Wiley \& Sons; 2009.

36. Farris DJ, Sawicki GS. Linking the mechanics and energetics of hopping with elastic ankle exoskeletons. J. Appl. Physiol. 2012; 113:1862-1872. [PubMed: 23065760]

37. Sawicki GS, Ferris DP. Mechanics and energetics of level walking with powered ankle exoskeletons. J. Exp. Biol. 2008; 211:1402-1413. [PubMed: 18424674]

38. Donelan JM, Kram R, Kuo AD. Simultaneous positive and negative external mechanical work in human walking. J. Biomech. 2002; 35:117-124. [PubMed: 11747890]

39. Brockway JM. Derivation of formulae used to calculate energy expenditure in man. Hum. Nut. Clin. Nut. 1987; 41:463-471.

40. Glantz, SA. Primer of Biostatistics. New York, NY, USA: McGraw-Hill; 2005.

41. Tucker VA. The Energetic Cost of Moving About: Walking and running are extremely inefficient forms of locomotion. Much greater efficiency is achieved by birds, fish-and bicyclists. Amer. Scientist. 1975; 63:413-419. [PubMed: 1137237]

42. Donelan JM, et al. Biomechanical energy harvesting: generating electricity during walking with minimal user effort. Science. 2008; 319:807-810. [PubMed: 18258914]

43. Collins SH, Kuo AD. Recycling energy to restore impaired ankle function during human walking. PLoS ONE. 2010; 5:e9307. [PubMed: 20174659] 
a

C

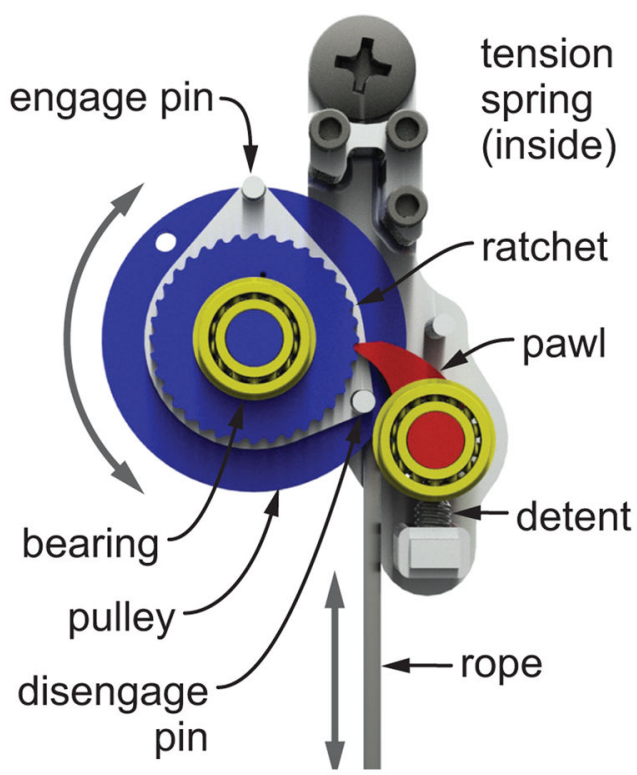

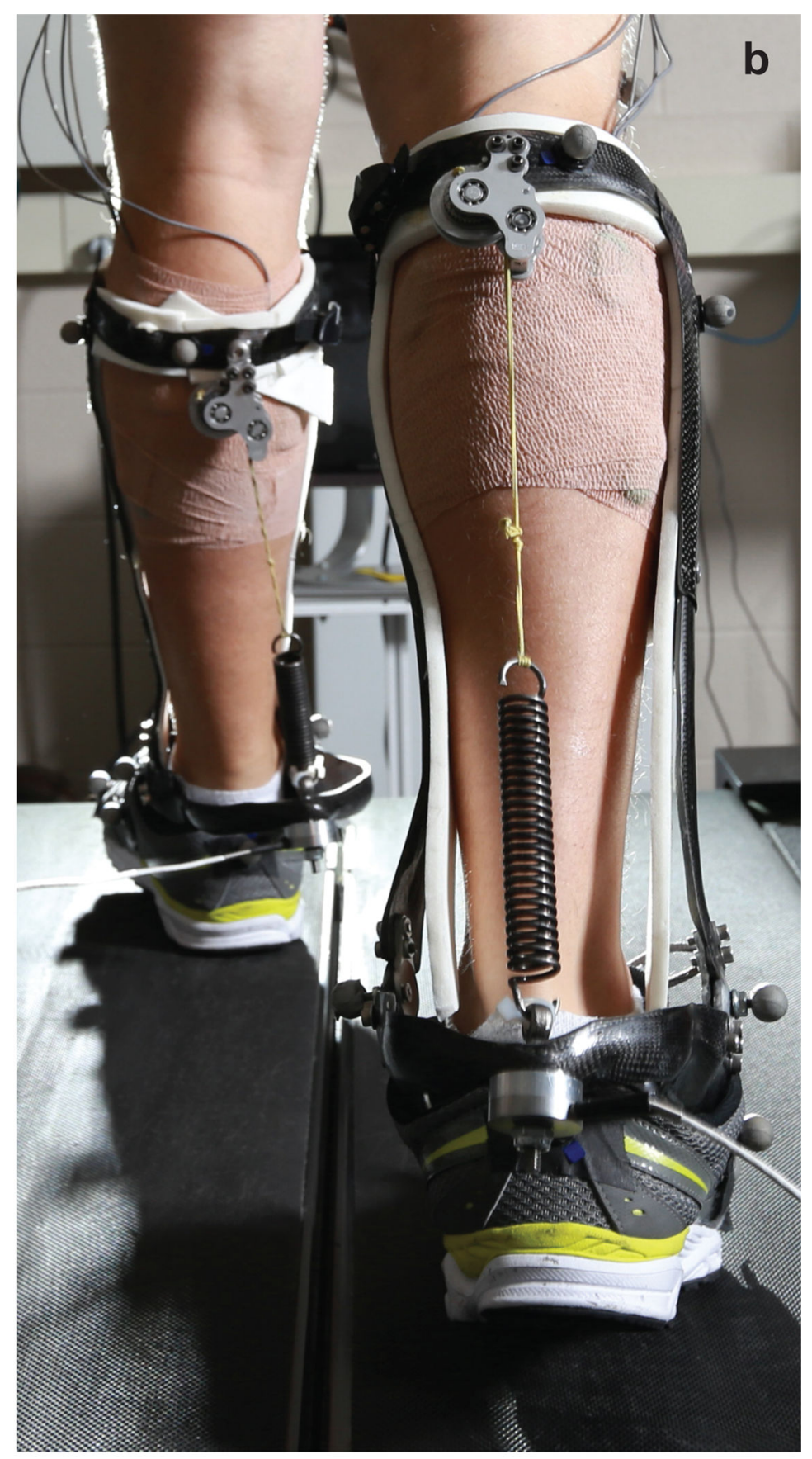

Figure 1. Unpowered exoskeleton design

(a) The exoskeleton comprises rigid sections attached to the human shank and foot and hinged at the ankle. A passive clutch mechanism and series spring act in parallel with the calf muscles and Achilles tendon. (b) Participant walking with the device. Load cells measured spring force. Photo by Stephen Thrift. (c) The passive clutch mechanism has no electronics, but instead uses a ratchet and pawl that mechanically engage the spring when the foot is on the ground and disengage it when the foot is in the air. 


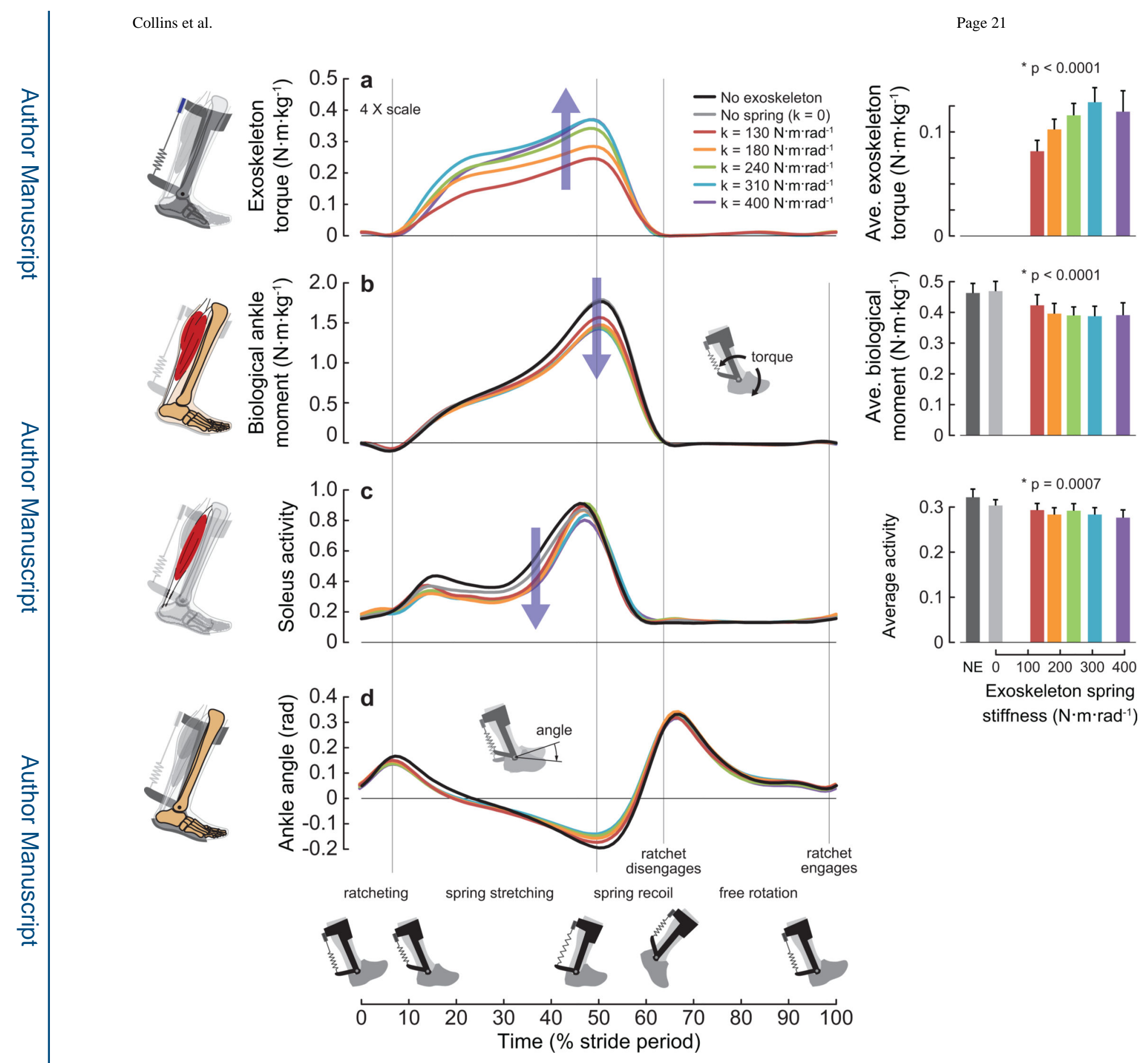

Figure 2. Mechanics and muscle activity

(a) Exoskeleton torque (normalized to body mass) in time (normalized to stride period) for each spring, averaged across participants. Bars at right are the average of these trajectories in time, with error bars denoting standard error and p-values indicating the results of ANOVA tests for an effect of spring stiffness. Exoskeleton torque increased with spring stiffness (except with the stiffest spring, which tended to be engaged later in stance). (b) Time course of the biological contributions to ankle moment, which decreased with increasing spring stiffness. (c) Time course of electrical activity in the soleus muscle, an 
ankle plantarflexor, which decreased with increasing spring stiffness. (d) Time course of ankle joint angle, which triggered passive clutch engagement and disengagement. The ratchet was engaged at heel strike, took up slack through foot flat, held the spring as it stretched and recoiled through mid and late stance, and disengaged to allow toe clearance during leg swing. The average stride period was $1.15 \pm 0.08 \mathrm{~s}$ (mean \pm s.d.). 


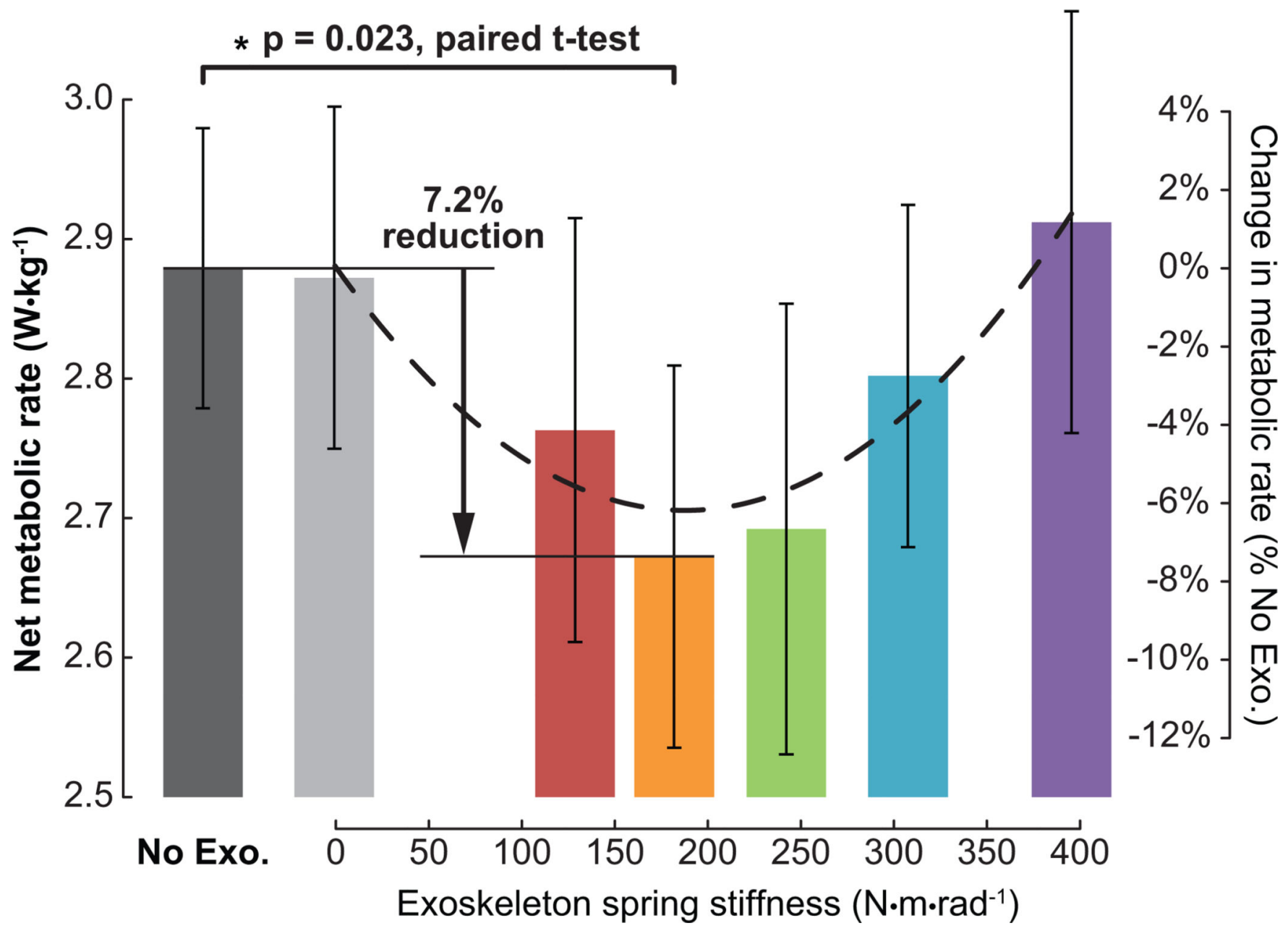

Figure 3. Human metabolic rate

Spring stiffness affected metabolic rate $[\mathrm{N}=9$; ANOVA with second order model; $\mathrm{p}($ stiffness $)=0.016, \mathrm{p}\left(\right.$ stiffness $\left.\left.^{2}\right)=0.008\right]$. Net metabolic rate, with the value for quiet standing subtracted out, was $7.2 \pm 2.6 \%$ (mean \pm s.e.m.) lower with the $180 \mathrm{~N} \cdot \mathrm{m} \cdot \mathrm{rad}^{-1}$ spring (orange bar) than during normal walking (dark gray bar; paired two-sided t-test with correction for multiple comparisons; $p=0.023$ ). The dashed line is a quadratic best fit to mean data from exoskeleton conditions $\left(\mathrm{R}^{2}=0.91, \mathrm{p}=0.029\right)$. Wearing the exoskeleton with the spring removed (light gray bar, $\mathrm{k}=0$ ) did not increase energy cost compared to normal walking (paired t-test; $\mathrm{p}=0.9$ ). Error bars depict standard error, dominated by intersubject variability. 